\title{
General features of Nash equilibria in combinations of elementary interactions in symmetric two-person games
}

\author{
György Szabóa $^{(\mathbb{D})}$ and Balázs Király ${ }^{\mathrm{b}}$ \\ Institute for Technical Physics and Material Sciences, Centre for Energy Research, P. O. Box 49, 1525 Budapest, Hungary
}

Received 27 April 2021 / Accepted 2 May 2021 / Published online 17 May 2021

(C) The Author(s) 2021

\begin{abstract}
Two-person games are used in many multi-agent mathematical models to describe pair interactions. The type (pure or mixed) and the number of Nash equilibria affect fundamentally the macroscopic behavior of these systems. In this paper, the general features of Nash equilibria are investigated systematically within the framework of matrix decomposition for $n$ strategies. This approach distinguishes four types of elementary interactions that each possess fundamentally different characteristics. The possible Nash equilibria are discussed separately for different types of interactions and also for their combinations. A relation is established between the existence of infinitely many mixed Nash equilibria and the zero-eigenvalue eigenvectors of the payoff matrix.
\end{abstract}

\section{Introduction}

The traditional concepts of game theory [1] have been extensively used for a long time in quantitative investigations of phenomena that occur in biological and social systems [2-6]. Pair interactions between the players are generally described using the formalism of two-player symmetric games, in which pure strategies can represent different physical states, forms of human behavior, or biological species. The applicability of methods developed in statistical physics to the mathematical consideration of the macroscopic behavior of these systems aroused the interest of physicists [7-13]. Most of the recent efforts are focused on the suppression of social dilemmas and the exploration of the consequences of cyclic dominance.

In game theory, selfish and intelligent players wish to maximize their own income [1]. In so-called noncooperative games players must choose their strategy without communicating with each other. In these systems, Nash equilibria [14,15], collective strategy profiles from which unilateral deviation is not beneficial, are considered as solutions. Nash proved the existence of at least one Nash equilibrium when the players are allowed to use mixed strategies, which randomly select an available strategy according to some probabilities. One of the main problems in game theory is related to the number of Nash equilibria because the existence of two or more solutions causes difficulties for the players in the absence of communication. These features are inherited by evolutionary games whose interactions are

\footnotetext{
a e-mail: szabo@mfa.kfki.hu (corresponding author)

be-mail: kiralyb@mfa.kfki.hu
}

generally built up from two-person symmetric games $[8-12,16]$.

Some properties of evolutionary games can be predicted just based on their Nash equilibria. Small variations in the payoff parameters usually do not cause relevant changes in the behavior of a game, so the classification of possible interaction types and their general features has been the subject of a wide range of systematic scientific investigations. For example, the average number of Nash equilibria was studied by Berg $[17,18]$ in $n$-strategy games with payoff elements determined by random numbers, and the introduction of potential games [19-21] has initiated the analysis of the decomposition of games [22-28].

In the simplest non-cooperative game there are two equivalent players with two available strategies and their strategy-dependent income is defined by a payoff matrix of four possible payoff elements. In these games, the search for the Nash equilibria can be simplified by exploiting two features, namely that the rank of incomes is not affected if the matrix elements are multiplied by a positive number and shifted by a constant. Consequently, this type of game can be defined by two independent parameters. The corresponding two-dimensional map of the possible Nash equilibria distinguishes four characteristic types of behavior illustrated generally by the traditional "prisoner's dilemma", "chicken" or "snow-drift", "staghunt", and "harmony" or "trivial" games. These games are well discussed in the above-mentioned textbooks and reviews. This simple method, however, cannot be easily applied to three or more strategies because of the large number of payoff parameters [29]. Instead of it, analyses are generally restricted to $n$-strategy games defined by just a few parameters. 
The above-mentioned reduction of the number of relevant parameters can be interpreted as a type of equivalence between games. This concept can be extended by recognizing that two games can be transformed into each other by relabelling the strategies [30,31]. This type of isomorphism shrinks the range of possible behavior.

Here we discuss Nash equilibria within the framework of matrix decomposition [28,32]. In this approach, the $n$-strategy payoff matrix is built up from four types of elementary matrices that represent fundamentally different interactions in evolutionary games. The existence of pure and mixed Nash equilibria are discussed for these elementary interactions and also for some characteristic combinations. Isomorphic games can be easily identified within the classes of elementary interactions. Additionally, we briefly describe some methods that can be used to determine Nash equilibria.

\section{Fundamental concepts and a brief survey of previous results}

The relevant concepts of game theory [1] can be surveyed briefly if we restrict ourselves to non-cooperative two-person symmetric games. In this family of games, there are two intelligent and selfish players $(X$ and $Y$ ) who do not communicate with each other. They both have $n$ options (called strategies) to choose from, and they both wish to make a choice that maximizes their own utility. This utility or payoff for a given strategy pair is defined by an $n \times n$ payoff matrix $\mathbf{A}$. The entry $A_{j k}(1 \leq j, k \leq n)$ defines the payoff of player $X$ if she chooses the $j$-th strategy while her co-player $Y$ chooses the $k$-th strategy. In symmetric games the players are equivalent, they receive the same payoff when they choose the same strategy, and their payoffs are exchanged when their strategies are exchanged.

The players may be allowed to use mixed strategies, which can be represented by $n$-dimensional vectors as

$$
\mathbf{s}_{x}=\left(\begin{array}{c}
x_{1} \\
x_{2} \\
\vdots \\
x_{n}
\end{array}\right) \quad \text { and } \quad \mathbf{s}_{y}=\left(\begin{array}{c}
y_{1} \\
y_{2} \\
\vdots \\
y_{n}
\end{array}\right)
$$

where the vector components quantify the probability of choosing the corresponding strategy, thus

$$
x_{j}, y_{j} \geq 0 \forall j \text { and } \sum_{j} x_{j}=\sum_{j} y_{j}=1 .
$$

In this notation, pure strategies are represented by Cartesian basis vectors, which also denote the vertices of the simplex defined by the previous conditions. Using this notation, the (expected) payoffs $\left(U_{x}\right.$ and $\left.U_{y}\right)$ of the players are given by

$$
U_{x}=\mathbf{s}_{x} \cdot \mathbf{A} \mathbf{s}_{y} \text { and } U_{y}=\mathbf{s}_{y} \cdot \mathbf{A} \mathbf{s}_{x} .
$$

In these games, a Nash equilibrium is a strategy pair $\left(\mathbf{s}_{x}^{\star}, \mathbf{s}_{y}^{\star},\right)$ whose payoffs cannot be improved by unilateral strategy changes, that is,

$$
\mathbf{s}_{x}^{\star} \cdot \mathbf{A} \mathbf{s}_{y}^{\star} \geq \mathbf{s}_{x}^{\prime} \cdot \mathbf{A} \mathbf{s}_{y}^{\star} \text { and } \mathbf{s}_{y}^{\star} \cdot \mathbf{A} \mathbf{s}_{x}^{\star} \geq \mathbf{s}_{y}^{\prime} \cdot \mathbf{A} \mathbf{s}_{x}^{\star},
$$

where $\mathbf{s}_{x}^{\prime} \neq \mathbf{s}_{x}^{\star}$ and $\mathbf{s}_{y}^{\prime} \neq \mathbf{s}_{y}^{\star}$.

John Nash proved the existence of such equilibrium points $[14,15]$. However, we have to emphasize that difficulties can still arise if a non-cooperative game has more than one Nash equilibrium. In these cases, the absence of communication and trust can prevent the players from finding the optimal solution. Furthermore, even single Nash equilibria are not always optimal. They can create social dilemmas when selfishness dictates strategy choices that lead to lower payoffs than what cooperation could provide. (This happens in, for example, prisoner's dilemma, donation, and snowdrift games.)

Game theory textbooks [1,33-38] describe a variety of methods for finding Nash equilibria. Here, we recall the flow graph method for finding pure Nash equilibria. This method constructs a directed network whose nodes correspond to the possible strategy pairings similar to the elements of the payoff matrix. The nodes are connected according to unilateral strategy changes on the analogy of the dynamical graph introduced by Schnakenberg [39], and the edges point toward the strategy pairing preferred by the active player. In this flow graph, pure Nash equilibria have only incoming edges. This approach is equivalent to the traditional method of checking the conditions (4) row by row and column by column. At the same time, the flow graph visualizes directed loops, which prevent the game from having a potential, a quantity whose properties we will detail later. This approach can be applied to those payoff matrices that have different values in each row and column.

The topological features of the flow graph remain unchanged if the payoff matrix is multiplied by a positive number or weakly perturbed. However, multiplying the payoff matrix by a negative number reverses the direction of all edges of the flow graph.

Figure 1 shows the flow graph of a three-strategy symmetric game that has three pure Nash equilibria. This game illustrates a situation in which intelligent players cannot decide which strategy to choose because all of the game's Nash equilibria are Pareto optimal, that is, neither player's income can be increased without decreasing the other player's payoff $[8,40,41]$. This example specifically and the flow graph method in general highlight that only one pure Nash equilibrium can exist in each row and column of the payoff matrix, which caps the number of pure Nash equilibria at $n$ in an $n$ strategy game. Unlike the game in Fig. 1, not all games have a full complement of pure Nash equilibria, and some have none at all. The best known example of a game without pure Nash equilibria is the three-strategy rock-paper-scissors game. 


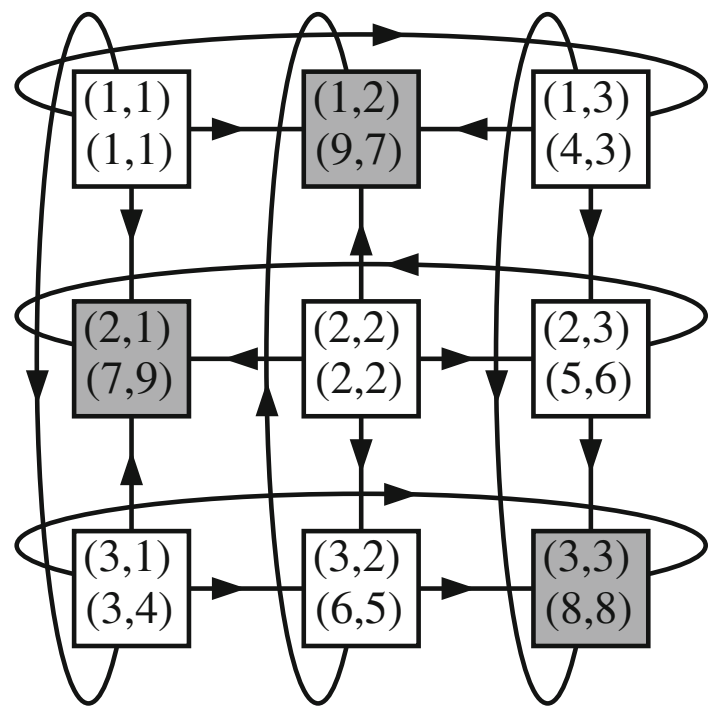

Fig. 1 The flow graph of a three-strategy symmetric game. The upper number pairs inside its nodes (the square boxes) refer to the strategy choices of the players $X$ and $Y$, while the lower pairs define their corresponding payoffs. Gray boxes indicate Nash equilibria

The standard method for finding mixed Nash equilibria assumes that both players wish to maximize their payoff by suitably varying their probability parameters, so that at the equilibrium point

$$
\frac{\partial U_{x}}{\partial x_{j}}=0 \text { and } \frac{\partial U_{y}}{\partial y_{j}}=0 \forall j
$$

subject to the conditions (2). Under the assumptions that the payoff matrix is invertible and the solution belongs to the interior of the simplex defined by Eq. (2), a straightforward calculation yields a single, symmetric possible mixed Nash equilibrium, namely,

$$
\mathbf{s}_{x}^{\star}=\mathbf{s}_{y}^{\star}=\frac{1}{\mathcal{C}} \mathbf{A}^{-1} \mathbf{u},
$$

where $\mathbf{A}^{-1}$ is the inverse matrix of $\mathbf{A}, \mathbf{u}$ is the all-one vector $\left(u_{j}=1 \forall j\right)$, and $\mathcal{C}$ is a normalization factor. This equilibrium strategy coincides with the second player's (also interior) optimal solution in the zero-sum game defined by $\mathbf{A}$ [42-44]. Eq. (5) gives the same prediction if $\mathbf{A}$ is replaced by $\alpha \mathbf{A}$.

For example, the payoff matrix and its inverse for the game in Fig. 1 are:

$$
\mathbf{A}=\left(\begin{array}{lll}
1 & 9 & 4 \\
7 & 2 & 5 \\
3 & 6 & 8
\end{array}\right) \text { and } \mathbf{A}^{-1}=\frac{1}{239}\left(\begin{array}{ccc}
14 & 48 & -37 \\
41 & 4 & -23 \\
-36 & -21 & 61
\end{array}\right)
$$

The resulting mixed Nash equilibrium is

$$
\mathbf{s}_{x}^{\star}=\mathbf{s}_{y}^{\star}=\frac{1}{51}\left(\begin{array}{c}
25 \\
22 \\
4
\end{array}\right),
$$

from which the players derive the same expected payoff $\left(U_{x}=U_{y}=\frac{239}{51} \approx 4.686\right)$, which is smaller than those provided by the pure Nash equilibria. Intelligent players should conclude that without communication, fraternity, or trust they cannot select one of the three pure Nash equilibria.

Additional, sometimes curious Nash equilibria can also exist beside the one predicted by Eq. (6), as we will show in the next section that details the framework of matrix decomposition.

\section{Nash equilibria of elementary interactions}

On the analogy of vectors, the payoff matrix $\mathbf{A}$ can also be built up as a linear combination of a set of basis matrices. The simplest way to do this is to construct $\mathbf{A}$ from $n \times n$ Cartesian type basis matrices that each contain a single 1 while all of their other entries equal 0 . Instead of this, we could choose other sets of basis matrices, for example, created by Fourier transformation [27] or dyadic products [28]. Here, we use a framework [32] that distinguishes four orthogonal types of elementary interaction, namely, games with self- $\left(\mathbf{A}^{(\mathrm{se})}\right)$ and cross-dependent $\left(\mathbf{A}^{(\mathrm{cr})}\right)$ payoffs, coordination-type $\left(\mathbf{A}^{(\mathrm{co})}\right)$ and cyclic-type $\left(\mathbf{A}^{\text {(cyc) }}\right)$ interactions. Two matrices ( $\mathbf{A}$ and $\mathbf{B}$ ) are orthogonal if $\sum_{j, k} A_{j k} B_{j k}=0$.

In this notation, the payoff matrix is given as

$$
\mathbf{A}=\mathbf{A}^{(\mathrm{av})}+\mathbf{A}^{(\mathrm{se})}+\mathbf{A}^{(\mathrm{cr})}+\mathbf{A}^{(\mathrm{co})}+\mathbf{A}^{(\mathrm{cyc})},
$$

where $\mathbf{A}^{(a v)}$ defines the irrelevant term of the decomposition that is proportional to the all-one matrix $\mathbf{A}^{(\mathrm{u})}$ :

$$
\mathbf{A}^{(\mathrm{av})}=a \mathbf{A}^{(\mathrm{u})}=a\left(\begin{array}{cccc}
1 & 1 & \ldots & 1 \\
1 & 1 & \ldots & 1 \\
\vdots & \vdots & \ddots & \vdots \\
1 & 1 & \ldots & 1
\end{array}\right) \text {, where } a=\frac{1}{n^{2}} \sum_{j, k} A_{j k}
$$

Notice that $a$ is the average value of the entries of the payoff matrix. If $\mathbf{A}=\mathbf{A}^{\text {(av) }}$, then the payoff is independent of the strategy choices and any strategy pair satisfies the conditions (4). In other words, this game has infinitely many Nash equilibria.

\subsection{The cross- and self-dependent components}

The cross-dependent component $\left(\mathbf{A}^{(\mathrm{cr})}\right)$ is defined by a matrix with row-independent payoffs in each column, that is,

$$
\mathbf{A}^{(\mathrm{cr})}=\left(\begin{array}{cccc}
\gamma_{1} & \gamma_{2} & \ldots & \gamma_{n} \\
\gamma_{1} & \gamma_{2} & \ldots & \gamma_{n} \\
\vdots & \vdots & \ddots & \vdots \\
\gamma_{1} & \gamma_{2} & \ldots & \gamma_{n}
\end{array}\right), \text { where } \gamma_{k}=\frac{1}{n} \sum_{j} A_{j k}-a
$$


This type of interaction is characterized by $(n-1)$ independent parameters because $\sum_{k} \gamma_{k}=0$. This condition ensures orthogonality between $\mathbf{A}^{(\mathrm{av})}$ and $\mathbf{A}^{(\mathrm{cr})}$. These two components do not affect the payoff variation of player $X$ (or $Y$ ) if she modifies her strategy unilaterally. Thus, if $\mathbf{A}=\mathbf{A}^{(a v)}+\mathbf{A}^{(c r)}$, then any strategy pair is a non-strict Nash equilibrium.

The self-dependent component $\left(\mathbf{A}^{(\mathrm{se})}\right)$ is defined by a matrix of identical, column-independent elements in its rows, that is,

$$
\mathbf{A}^{(\mathrm{se})}=\left(\begin{array}{cccc}
\varepsilon_{1} & \varepsilon_{1} & \ldots & \varepsilon_{1} \\
\varepsilon_{2} & \varepsilon_{2} & \ldots & \varepsilon_{2} \\
\vdots & \vdots & \ddots & \vdots \\
\varepsilon_{n} & \varepsilon_{n} & \ldots & \varepsilon_{n}
\end{array}\right), \text { where } \varepsilon_{j}=\frac{1}{n} \sum_{k} A_{j k}-a .
$$

Since $\sum_{j} \varepsilon_{j}=0, \mathbf{A}^{(\mathrm{se})}$ is also defined by $(n-1)$ independent parameters. In these games, the income of the players is not affected by their opponent's choice, so they are both motivated to choose the strategy with the highest $\varepsilon_{j}$. If the maximum is $l$-fold degenerate, then the game has a continuously infinite number of mixed Nash equilibria, which combine the highest-paying strategies with arbitrary probabilities. For instance, if a three-strategy self-dependent game is given by $\varepsilon_{1}=\varepsilon_{2}=1$ and $\varepsilon_{3}=-2$, then the players receive the highest income $\left(U_{x}=U_{y}=1\right)$ in mixed Nash equilibria of the form $\mathbf{s}_{x}^{+}=\left(x_{1}, 1-x_{1}, 0\right)$ and $\mathbf{s}_{y}^{+}=\left(y_{1}, 1-y_{1}, 0\right)\left(0 \leq x_{1}, y_{1} \leq 1\right)$.

Social dilemmas can occur in combinations of selfand cross-dependent components. The pure Nash equilibria of these games are solely determined by $\mathbf{A}^{\text {(se) }}$ while the payoff values are affected by $\mathbf{A}^{(\mathrm{cr})}$ too. The two-strategy donation game has this structure as well, and thus, the general $\mathbf{A}^{(\mathrm{cr})}+\mathbf{A}^{(\mathrm{se})}$ game can be considered as the $n$-strategy version of the donation game [32].

\subsection{The coordination component}

The coordination component is the symmetric part of $\mathbf{A}-\mathbf{A}^{(\mathrm{av})}-\mathbf{A}^{(\mathrm{cr})}-\mathbf{A}^{(\mathrm{se})}$ and it can be built up from elementary interactions $\mathbf{Q}^{(\mathrm{j}, \mathrm{k})}$ as

$$
\mathbf{A}^{(\mathrm{co})}=\sum_{\substack{j, k \\ j<k}} \nu_{j k} \mathbf{Q}^{(\mathrm{j}, \mathrm{k})},
$$

where $\mathbf{Q}^{(\mathrm{j}, \mathrm{k})}$ has only four non-zero elements, namely, $Q_{j j}^{(\mathrm{j}, \mathrm{k})}=Q_{k k}^{(\mathrm{j}, \mathrm{k})}=-Q_{j k}^{(\mathrm{j}, \mathrm{k})}=-Q_{k j}^{(\mathrm{j}, \mathrm{k})}=1$. The whole coordination component is given by $n(n-1) / 2$ parameters $\nu_{j k}$ that quantify the strength of coordination (or Ising-type interaction) between all possible different strategy pairs $(j<k)$.

When the payoff matrix consists of just one elementary coordination component (i.e., $\mathbf{A}=\nu_{j k} \mathbf{Q}^{(\mathrm{j}, \mathrm{k})}$ ), then the game has two equivalent pure Nash equilibria, namely, the strategy pairs $(j, j)$ and $(k, k)$ if $\nu_{j k}$ is positive and $(j, k)$ and $(k, j)$ if $\nu_{j k}$ is negative.
If all $\nu_{j k}$ are positive, then $\mathbf{A}^{(\text {co) }}$ has $n$ pure Nash equilibria because all of its offdiagonal elements are negative, while $A_{j j}^{(\mathrm{co})}>0 \forall j$. In this case, the players do best if they choose the symmetric strategy pair $(l, l)$ with the highest corresponding matrix element, $A_{l l}=\max \left(A_{j j}^{(\mathrm{co})}\right)$.

In potential games $[19,23,45]$, that is, games without a cyclic component (i.e., $\mathbf{A}^{(\mathrm{cyc})}=0$ ), a more general version of this rule applies: The highest-paying Nash equilibrium is determined by the highest entry in the game's symmetric potential matrix $\mathbf{V}$, which is given by

$$
\mathbf{V}=\mathbf{A}^{(\mathrm{se})}+\mathbf{A}^{(\mathrm{se}) T}+\mathbf{A}^{(\mathrm{co})}+\mathbf{A}^{(\mathrm{av})} .
$$

It is emphasized that the symmetric two-strategy games are potential games that simplifies their analyses. Furthermore, if $\max \left(V_{j k}\right)=V_{l l}$ is a diagonal entry, then the best choice for the players is the corresponding symmetric strategy pair $(l, l)$, whereas if the interaction is dominated by an anti-coordination component $\left(\max \left(V_{j k}\right)=V_{l m}=V_{m l}\right)$ than the game has two equivalent pure Nash equilibria, namely, the strategy pairs $(l, m)$ and $(m, l)$.

If the game is of the form $\mathbf{A}=\mathbf{A}^{(\mathrm{av})}+\mathbf{A}^{(\mathrm{cr})}+\mathbf{A}^{(\mathrm{co})}$, then the two players receive the same income in Nash equilibria, which means that their individual and common interests coincide. Changing the cross-dependent component modifies neither the potential $\mathbf{V}$ nor the Nash equilibria. At the same time, it does change the payoffs, which may lead to a social dilemma. Previous investigations show that different kinds of social dilemmas can be observed in large domains of the $n^{2}$ dimension parameter space of two-player games [32].

Due to its orthogonality to $\mathbf{A}^{(\mathrm{av})}, \mathbf{A}^{\text {(cr) }}$, and $\mathbf{A}^{(\mathrm{se})}$ the sum of the entries of $\mathbf{A}^{(\mathrm{co})}$ is zero in each of its rows and columns. This feature guarantees that if player $Y$ chooses her strategy at random, then player $X$ 's expected income is zero regardless of his strategy choice. Thus, the strategy pair $\mathbf{s}_{x}^{\star}=\mathbf{s}_{y}^{\star}=\frac{1}{n} \mathbf{u}$ is a mixed Nash equilibrium for coordination-type $\mathbf{A}=\mathbf{A}^{\text {(co) }}$ games. Notice that this argument hinges on $\mathbf{A}^{(\mathrm{co})} \mathbf{u}=\mathbf{0}$, that is, $\mathbf{u}$ being the eigenvector of $\mathbf{A}^{(\mathrm{co})}$ with zero eigenvalue. This means that any other eigenvectors of $\mathbf{A}^{(\mathrm{co})}$ with zero eigenvalue also constitute mixed Nash equilibria. For example, a four-strategy coordination game composed of two non-overlapping elementary coordination games has the payoff matrix

$$
\mathbf{A}^{(\mathrm{co})}=\left(\begin{array}{cccc}
\nu_{12} & -\nu_{12} & 0 & 0 \\
-\nu_{12} & \nu_{12} & 0 & 0 \\
0 & 0 & \nu_{34} & -\nu_{34} \\
0 & 0 & -\nu_{34} & \nu_{34}
\end{array}\right)
$$

which has mixed Nash equilibria with zero eigenvalue given by

$$
\mathbf{s}_{x}=\left(\begin{array}{c}
x \\
x \\
0.5-x \\
0.5-x
\end{array}\right) \quad \text { and } \quad \mathbf{s}_{y}=\left(\begin{array}{c}
y \\
y \\
0.5-y \\
0.5-y
\end{array}\right)
$$


where $0 \geq x, y \geq 0.5$. The four-state Ashkin-Teller model is the linear combination of three similar coordination components when $\nu_{12}=\nu_{34}, \nu_{13}=\nu_{24}$, and $\nu_{14}=\nu_{23}[46]$.

In light of the results above, we can deduce a general rule for finding mixed Nash equilibria in coordinationtype games. The strategies are naturally divided into disjunct groups along the lines of elementary interactions in such a way that strategies within each group are connected by a non-zero-strength elementary coordination component to at least one other strategy in the same group and the groups cannot be further divided without breaking at least one such connection. Strategies that are not connected to any other strategy (i.e., entries in their row and column of the payoff matrix equal zero) form one-strategy groups on their own. In mixed Nash equilibria with zero eigenvalue, strategies that belong to the same group are chosen with the same probability, and these probabilities may be different for different groups. This set of mixed Nash equilibria remains unchanged when varying the value of non-zero elementary coordination coefficients, that is, $\nu_{j k} \rightarrow \nu_{j k}^{\prime}$ provided that $\nu_{j k}, \nu_{j k}^{\prime} \neq 0$.

Coordination games with more eigenvectors with zero eigenvalue have further curious mixed Nash equilibria. For example, the matrix

$$
\mathbf{A}^{(\mathrm{co})}=\left(\begin{array}{cccc}
-1 & 1 & -1 & 1 \\
1 & -1 & 1 & -1 \\
-1 & 1 & -1 & 1 \\
1 & -1 & 1 & -1
\end{array}\right)
$$

is composed of four elementary anti-coordination and two elementary coordination games. This matrix's rank is reduced by three eigenvectors with zero eigenvalue. Notice that either the sum or the difference of any two rows (or columns) is zero. For this four-strategy game the set of mixed Nash equilibria can be given as

$$
\mathbf{s}_{x}^{\star}=\left(\begin{array}{c}
0.25+x_{1} \\
0.25+x_{2} \\
0.25-x_{1} \\
0.25-x_{2}
\end{array}\right) \quad \text { and } \quad \mathbf{s}_{y}^{\star}=\left(\begin{array}{c}
0.25+y_{1} \\
0.25+y_{2} \\
0.25-y_{1} \\
0.25-y_{2}
\end{array}\right)
$$

where $0 \geq x_{1}, x_{2}, y_{1}, y_{2} \geq 0.25$.

Equation (17) defines a game in which $\mathbf{A}^{(\mathrm{co})}$ remains unchanged under cyclic permutations of the strategy labels. Games with a similar symmetry can be constructed for any $n$. This symmetry can be exploited in the calculation of eigenvalues and eigenvectors. We illustrate this method on a five-strategy game defined by elementary coordination components of two strengths $(1$ and $\nu)$ as

$$
\mathbf{A}^{(\operatorname{co5})}=\left(\begin{array}{ccccc}
2+2 \nu & -1 & -\nu & -\nu & -1 \\
-1 & 2+2 \nu & -1 & -\nu & -\nu \\
-\nu & -1 & 2+2 \nu & -1 & -\nu \\
-\nu & -\nu & -1 & 2+2 \nu & -1 \\
-1 & -\nu & -\nu & -1 & 2+2 \nu
\end{array}\right)
$$

The eigenvectors of this matrix are the real and imaginary parts of the complex Fourier components $\mathbf{v}(q)$ defined by

$$
\mathbf{v}(q)=\left(\begin{array}{c}
e^{\mathrm{i} \varphi q} \\
e^{\mathrm{i} \varphi q 2} \\
e^{\mathrm{i} \varphi q 3} \\
e^{\mathrm{i} \varphi q 4} \\
1
\end{array}\right), \text { or } v_{j}(q)=e^{\mathrm{i} \varphi q j}
$$

where $\varphi=\frac{2 \pi}{5}$ and the possible values of the wave number are $q=0, \pm 1, \pm 2$. A similar method is used in solid state physics (see textbooks, e.g., [47,48]) when determining the spectrum of lattice vibrations on a onedimensional lattice. The eigenvalue $\omega(q)$ is given by

$$
\omega(q) \mathbf{v}(q)=\mathbf{A}^{(\operatorname{co} 5)} \mathbf{v}(q)
$$

A straightforward calculation yields

$$
\omega(q)=-4 \sin ^{2} \frac{\varphi q}{2}\left[1+4 \nu \cos ^{2} \frac{\varphi q}{2}\right] .
$$

This eigenvalue is zero for $q=0$ and the corresponding eigenvector defines the uniformly random mixed Nash equilibrium. If $\nu$ is such that the twofold degenerate eigenvalues equal 0 for some $q$, that is, $\omega(q)=\omega(-q)=0$, then an additional degree of freedom opens up in the space of zero-eigenvalue Nash equilibria. Specifically, $\omega(1)=\omega(-1)=0$ if $\nu=-1 / 4 \cos ^{2}(\varphi / 2)$ and the corresponding Nash equilibria can be written as

$$
\mathbf{s}_{x}^{\star}=\frac{1}{5}\left(\begin{array}{c}
1 \\
1 \\
1 \\
1 \\
1
\end{array}\right)+c_{1}\left(\begin{array}{c}
\cos (\varphi) \\
\cos (2 \varphi) \\
\cos (3 \varphi) \\
\cos (4 \varphi) \\
1
\end{array}\right)+c_{2}\left(\begin{array}{c}
\sin (\varphi) \\
\sin (2 \varphi) \\
\sin (3 \varphi) \\
\sin (4 \varphi) \\
0
\end{array}\right)
$$

and

$$
\mathbf{s}_{y}^{\star}=\frac{1}{5}\left(\begin{array}{l}
1 \\
1 \\
1 \\
1 \\
1
\end{array}\right)+c_{1}^{\prime}\left(\begin{array}{c}
\cos (\varphi) \\
\cos (2 \varphi) \\
\cos (3 \varphi) \\
\cos (4 \varphi) \\
1
\end{array}\right)+c_{2}^{\prime}\left(\begin{array}{c}
\sin (\varphi) \\
\sin (2 \varphi) \\
\sin (3 \varphi) \\
\sin (4 \varphi) \\
0
\end{array}\right)
$$

where the possible values of $c_{1}, c_{2}, c_{1}^{\prime}$, and $c_{2}^{\prime}$ are limited by the constraints in Eq. (2). When $\nu=$ $-1 / 4 \cos ^{2} \varphi$ and $\omega(2)=\omega(-2)=0$ instead, the set of resulting mixed Nash equilibria is described by a very similar formula, in which the uniformly random mixed Nash equilibrium is perturbed by the Fourier components belonging to $q= \pm 2$.

The same general method can be used to derive zero-eigenvalue Nash equilibria as a linear combination of Fourier components whenever the payoff matrix remains unchanged under the cyclic permutation of strategy labels. We have to emphasize, however, that cyclic permutation symmetry is not a nec- 
essary condition for the existence of additional zeroeigenvalue eigenvectors, because they always emerge in a coordination-type game if the rank of $\mathbf{A}^{(\mathrm{co})}$ is less than $(n-1)$.

Some curious coordination games that exhibit interesting features have already been used as pair interactions in evolutionary games [49]. Some variants of the four-state Ashkin-Teller model [46] present a wide variety of different critical behavior [50-52].

\subsection{The cyclic component}

The cyclic component is the antisymmetric part of $\mathbf{A}-\mathbf{A}^{(\text {av })}-\mathbf{A}^{(\mathrm{cr})}-\mathbf{A}^{(\mathrm{se})}$ and it can be built up from elementary cyclic interactions $\mathbf{C}^{(\mathrm{j}, \mathrm{k}, \mathrm{l})}(\mathrm{j}<\mathrm{k}<\mathrm{l})$, which are voluntary rock-paper-scissor games, that is, if one of the players chooses strategy $m \neq j, k, l$, then he effectively declines to play the game and both players receive zero payoff. For example, this means that the $n$-strategy $\mathbf{C}^{(1,2,3)}$ game extends the three-strategy rock-paper-scissors game (involving strategies 1, 2, and 3 ) by having additional zero-filled rows and columns in its payoff matrix in the following way

$$
\mathbf{C}^{(1,2,3)}=\left(\begin{array}{cccccc}
0 & 1 & -1 & 0 & \ldots & 0 \\
-1 & 0 & 1 & 0 & \ldots & 0 \\
1 & -1 & 0 & 0 & \ldots & 0 \\
0 & 0 & 0 & 0 & \ldots & 0 \\
\vdots & \vdots & \vdots & \vdots & \ddots & \vdots \\
0 & 0 & 0 & 0 & \ldots & 0
\end{array}\right)
$$

The payoff matrix of the other elementary cyclic games can be derived from this matrix by suitably exchanging its rows and columns. Elementary cyclic components are not all independent of each other [28,53,54]. There are, however, many subsets of all $\mathbf{C}^{(\mathrm{j}, \mathrm{k}, \mathrm{l})}$ whose elements span the whole space of cyclic games. For example, if one of the indices is fixed (e.g., $j=1$ ), then the remaining $(n-1)(n-2) / 2$ voluntary rock-paper-scissors games are suffcient to describe any cyclic component as

$$
\mathbf{A}^{(\mathrm{cyc})}=\sum_{\substack{j, k \\ 1<k<l}} \lambda_{1 k l} \mathbf{C}^{(1, \mathrm{k}, \mathrm{l})}
$$

where each coefficient $\lambda_{1 k l}$ defines the strength of the corresponding elementary cyclic component. This choice of the set of basis matrices is convenient because $A_{k l}^{\text {(cyc) }}=\lambda_{1 k l}$ if $1<k<l$. The elements of $\mathbf{A}^{\text {(cyc) }}$ in its first row and column can be expressed in terms of $\lambda_{1 k l}$ through the orthogonality conditions $\sum_{m} A_{m l}^{(\text {cyc })}=0 \quad \forall l$ and $\sum_{m} A_{k m}^{(\mathrm{cyc})}=0 \forall k$.

The linear combinations of the elementary cyclic components are zero-sum games. Thus, the players receive zero income if both choose the same strategy and $U_{x}=-U_{y}$ otherwise. In the latter case the loser would want to choose another strategy to switch the loser and winner roles between them or at least enforce zero income for both players.
These games have no strict pure Nash equilibria. In the corresponding flow graphs (see Fig. 1) the nodes have both outgoing and incoming edges. At the same time, the random mixed strategy pair $\left(\mathbf{s}_{x}^{\star}=\mathbf{s}_{y}^{\star}=\frac{1}{n} \mathbf{u}\right)$ is always a Nash equilibrium. Additional sets of mixed Nash equilibria can occur in cases similar to the ones already discussed for coordination games. That is, if the strategies can be split into disjunct groups without cyclic interactions between the members of different groups, then the game has an infinite set of mixed Nash equilibria in which the strategies within a group are chosen with the same probability. For example, if the game is defined by the matrix in Eq. (25) (i.e., $\mathbf{A}=\mathbf{C}^{(1,2,3)}$ ), then the set of mixed Nash equilibria is given by

$$
\mathbf{s}_{x}^{\star}=\left(\begin{array}{c}
x_{1} \\
x_{1} \\
x_{1} \\
x_{4} \\
\vdots \\
x_{n}
\end{array}\right) \quad \text { and } \quad \mathbf{s}_{y}^{\star}=\left(\begin{array}{c}
y_{1} \\
y_{1} \\
y_{1} \\
y_{4} \\
\vdots \\
y_{n}
\end{array}\right)
$$

where

$$
x_{j}, y_{j} \geq 0 \forall j \quad \text { and } \quad 3 x_{1}+\sum_{\substack{j \\ j>3}} x_{j}=3 y_{1}+\sum_{\substack{j \\ j>3}} y_{j}=1 .
$$

The above-mentioned mixed Nash equilibria are trivial eigenvectors of $\mathbf{A}^{\text {(cyc) }}$ with zero eigenvalue. Further curious Nash equilibria exist for those $\mathbf{A}^{\text {(cyc) }}$ that have additional eigenvectors with zero eigenvalue. To illustrate the features of these games we recall the modified version of the rock-paper-scissors-lizard-Spock game analyzed by Kang et al. [55] on a square lattice. In this five-strategy model the pair interaction is described by the matrix

$$
\mathbf{A}^{(\mathrm{rpslS})}=\left(\begin{array}{ccccc}
0 & \lambda & -1 & 1 & -\lambda \\
-\lambda & 0 & \lambda & -1 & 1 \\
1 & -\lambda & 0 & \lambda & -1 \\
-1 & 1 & -\lambda & 0 & \lambda \\
\lambda & -1 & 1 & -\lambda & 0
\end{array}\right)
$$

which remains unchanged when the strategy labels are permutated cyclically. Thus, for the determination of additional mixed Nash equilibria we can again apply the method we used to $\mathbf{A}^{(\mathrm{co5})}$ [see Eq. (19)] in the previous section. In this game the eigenvalue of the Fourier components is an imaginary function of the wave number $q(q=0, \pm 1, \pm 2)[56]$, namely

$$
\omega(q)=2 \mathrm{i}[\sin 2 \varphi q-\lambda \sin \varphi q] .
$$

For $q=0$ the eigenvalue is zero $(\omega(0)=0)$ independently of the value of $\lambda$ and the corresponding mixed Nash equilibrium dictates uniformly random strategy, that is, $\mathbf{s}_{x}^{\star}=\mathbf{s}_{y}^{\star}=\frac{1}{n} \mathbf{u}$. Additional eigenvectors with zero eigenvalue appear when the ratio of the two elementary cyclic component strengths is the golden ratio 
$\Phi=(\sqrt{5}-1) / 2$. Specifically, $\omega(1)=\omega(-1)=0$ if $\lambda=1 / \Phi$ and $\omega(2)=\omega(-2)=0$ if $\lambda=\Phi$. In the first case, the corresponding set of mixed Nash equilibria is equivalent to the one given by Eqs. (23) and (24). We have to emphasize, however, that the $\lambda=1 / \Phi$ and $\lambda=\Phi \mathbf{A}^{(\mathrm{rpslS})}$ games are actually isomorphic, because a suitable relabelling of the strategies $(1 \rightarrow 1,2 \rightarrow 4$, $3 \rightarrow 2,4 \rightarrow 5$, and $5 \rightarrow 3$ ) transforms their payoff matrices into each other up to a multiplicative factor $\Phi$.

Monte Carlo simulations of the many-player evolutionary version of these pair interactions on a square lattice with imitation-type dynamics indicated the appearance of huge fluctuations when $\lambda \simeq \Phi$, which lead to the extinction of two of the strategies in small systems $[55,56]$. Similar phenomena may also emerge in other systems with infinitely many mixed Nash equilibria.

\subsection{Combinations of elementary interactions}

Combinations of the coordination and cyclic components $\left(\mathbf{A}=\mathbf{A}^{(\mathrm{co})}+\mathbf{A}^{(\mathrm{cyc})}\right)$ inherit the symmetric uniformly random Nash equilibrium $\left(\mathbf{s}_{x}^{\star}=\mathbf{s}_{y}^{\star}=\frac{1}{n} \mathbf{u}\right)$, because they both fulfill the conditions $\sum_{k} A_{j k}=$ $\sum_{k} A_{k j}=0$. As the cross-dependent component does not affect individual preferences, the uniformly random Nash equilibrium is even preserved in games of the form $\left.\mathbf{A}=\mathbf{A}^{(\mathrm{co})}+\mathbf{A}^{(\mathrm{cyc})}+\mathbf{A}^{(\mathrm{cr})}\right)$. Combinations of $\mathbf{A}^{(\mathrm{co})}$ and $\mathbf{A}^{(\mathrm{cyc})}$ can also have infinitely many mixed Nash equilibria if they have more than one eigenvector with zero eigenvalue. For example, if $\mathbf{A}=\mathbf{A}^{(\mathrm{cos})}+\mathbf{A}^{(\mathrm{rsplS})}$ with $\nu=-1 / 4 \cos ^{2}(\varphi / 2)$ and $\lambda=\Phi$, then their shared Nash equilibria [defined by Eqs. (23) and (24)] carry over to the combination.

The conditions $\sum_{k} A_{j k}=0$ and $\sum_{k} A_{k j}=0$ preclude the existence of the inverse of the payoff matrix and thus the applicability of the formula in Eq. (6). The determinant of $\mathbf{A}$ is zero for these games because its rows (and columns) are not all linearly independent. Nevertheless, if a game defined by $\mathbf{A}=\mathbf{A}^{(\mathrm{co})}+\mathbf{A}^{\text {(cyc) }}$ is shifted by an irrelevant component, then the modified matrix may become invertible and the formula (6) can be used to predict a single mixed Nash equilibrium. The reader can easily check that if the payoff matrix of the rock-paper-scissors game is modified by an additional uniform constant $\left(\mathbf{A}=\mathbf{A}^{(\mathrm{rps})}+a \mathbf{A}^{(\mathrm{u})}\right)$ then $\mathbf{A}$ becomes invertible and plugging the inverse into Eq. (6) reproduces the well-known mixed Nash equilibrium.

The uniformly random symmetric Nash equilibrium may be distorted or eliminated in games of the form $\mathbf{A}=\mathbf{A}^{(\mathrm{co})}+\mathbf{A}^{(\mathrm{cyc})}+\mathbf{A}^{(\mathrm{se})}$. For these games, the determinant of $\mathbf{A}$ is zero, so its inverse does not exist, which invalidates formula (6). However, this problem may be circumvented by the previous trick of shifting the payoffs by a constant $\left(\mathbf{A} \rightarrow \mathbf{A}+a \mathbf{A}^{(\mathrm{u})}\right)$ if the rank of $\mathbf{A}$ is $(n-1)$. Otherwise, the game has a set of infinitely many mixed Nash equilibria just like the games discussed above.

\section{Summary and conclusions}

The concept of matrix (game) decomposition has allowed us to extract some general features of the Nash equilibria of two-player symmetric $n$-strategy games via the systematic investigation of the four types of elementary interaction and their combinations. The essence of games is missing if the payoff matrix consists only of a cross-dependent component and an irrelevant constant. In these interactions self-interest does not motivate the players to prefer any one of the strategies, and accordingly these games have a set of infinitely many Nash equilibria, because any strategy pair satisfies the conditions of (non-strict or weak) Nash equilibria in them.

Real interaction between the players is also missing if the payoff matrix includes only a self-dependent component. In this type of elementary "interaction" the players can choose the strategy that provides the highest (and the same) payoff to them independently of each other. This symmetric strategy pair is the only pure Nash equilibrium. Mixed Nash equilibria can occur if multiple strategies all award the same maximal payoff.

The Nash equilibria of combinations of self- and cross-dependent components are determined just by the self-dependent term, but the cross-dependent component still affects the payoffs, which may cause the payoff in the Nash equilibrium to be not optimal for the players, creating a social dilemma.[]

The coordination component summarizes the contributions of elementary coordinations (rewarding players based on whether they choose the same or opposite options) between all different strategy pairs. These are friendship games when the players receive the same income regardless of which strategy pair they choose. If the elementary components encourage the choice of the same strategy for all strategy pairs, then the coordination game has $n$ pure Nash equilibria. In spite of the large number of these Nash equilibria, intelligent players will evidently choose the symmetric strategy pair that provides the highest income. The pure Nash equuilibria of potential games, which combine coordination, cross-, and self-dependent components, are similarly easy to identify. However, if a potential game is dominated by a strong anticoordinated strategy pair, then it has two equivalent pure Nash equilibria, which poses another kind of dilemma for the players in the absence of communication.

Further inferences can be drawn from fact that the matrices of the four elementary interaction types are orthogonal to each other. It guarantees that uniform random choice is a mixed Nash equilibrium strategy for both the coordination component and the cyclic component (which does not have pure Nash equilibria), because it is a zero-eigenvalue eigenvector of coordination- and cyclic-type payoff matrices, and consequently their linear combinations. If a payoff matrix has multiple zero-eigenvalue eigenvectors, then the corresponding game has a set of infinitely many mixed Nash equilibria spanned by the linear combinations of these eigenvectors. We emphasize, however, that despite 
its seemingly general nature, matrices with this property still only comprise a zero-measure subset of all possible payoff matrices.

Funding Open access funding provided by ELKH Wigner Research Centre for Physics.

Data Availability Statement This manuscript has no associated data or the data will not be deposited. [Authors' comment:...].

Open Access This article is licensed under a Creative Commons Attribution 4.0 International License, which permits use, sharing, adaptation, distribution and reproduction in any medium or format, as long as you give appropriate credit to the original author(s) and the source, provide a link to the Creative Commons licence, and indicate if changes were made. The images or other third party material in this article are included in the article's Creative Commons licence, unless indicated otherwise in a credit line to the material. If material is not included in the article's Creative Commons licence and your intended use is not permitted by statutory regulation or exceeds the permitted use, you will need to obtain permission directly from the copyright holder. To view a copy of this licence, visit http://creativecomm ons.org/licenses/by/4.0/.

\section{References}

1. J. von Neumann, O. Morgenstern, Theory of Games and Economic Behaviour (Princeton University Press, Princeton, NJ, 1944)

2. J. Maynard Smith, Evolution and the Theory of Games (Cambridge University Press, Cambridge, 1982)

3. J. Hofbauer, K. Sigmund, The Theory of Evolution and Dynamical Systems (Cambridge University Press, Cambridge, 1988)

4. J.W. Weibull, Evolutionary Game Theory (MIT Press, Cambridge, MA, 1995)

5. M.A. Nowak, Evolutionary Dynamics (Harvard University Press, Cambridge, MA, 2006)

6. W.H. Sandholm, Population Games and Evolutionary Dynamics (MIT University Press, Cambridge, MA, 2010)

7. J. Miękisz, J. Phys. A: Math. Gen. 37, 9891 (2004)

8. G. Szabó, G. Fáth, Phys. Rep. 446, 97 (2007)

9. C.P. Roca, J.A. Cuesta, A. Sánchez, Phys. Life Rev. 6, 208 (2009)

10. E. Frey, Physica A 389, 4265 (2010)

11. J. Tanimoto, Fundamentals of Evolutionary Game Theory and its Applications (Springer, Tokyo, 2015)

12. M. Perc, J.J. Jordan, D.G. Rand, Z. Wang, S. Boccaletti, A. Szolnoki, Phys. Rep. 687, 1 (2017)

13. V. Capraro, M. Perc, J.R. Soc, Interface 18, 20200880 (2021)

14. J. Nash, Proc. Natl. Acad. Sci. USA 36, 48 (1950)

15. J. Nash, Ann. Math. 54, 286 (1951)

16. M.R. Arefin, K.M.A. Kabir, M. Jusup, H. Ito, J. Tanimoto, Sci. Rep. 10, 16092 (2020)

17. J. Berg, M. Engel, Phys. Rev. Lett. 81, 4999 (1998)

18. J. Berg, M. Weigt, Europhys. Lett. 48, 129 (1999)
19. D. Monderer, L.S. Shapley, Games Econ. Behav. 14, 124 (1996)

20. J. Hofbauer, J. Econ. Theor. 85, 1 (1999)

21. S. Morris, T. Ui, J. Econ. Theor. 124, 45 (2005)

22. M. Voorneveld, H. Norde, Games Econ. Behav. 19, 235 (1997)

23. W.H. Sandholm, Games Econ. Behav. 70, 446 (2010)

24. O. Candogan, I. Menache, A. Ozdaglar, P.A. Parrilo, Math. Oper. Res. 36, 474 (2011)

25. S.H. Hwang, L. Rey-Bellet, Computing Research Repository (CoRR) 2011, Article No. 1106.3552 (2011)

26. O. Candogan, A. Ozdaglar, P.A. Parrilo, Games Econ. Behav. 82, 66 (2013)

27. G. Szabó, K.S. Bodó, B. Allen, M.A. Nowak, Phys. Rev. E 90, 042811 (2014)

28. G. Szabó, K.S. Bodó, B. Allen, M.A. Nowak, Phys. Rev. E 92, 022820 (2015)

29. A. Rapoport, M. Guyer, Yearb Soc. Gen. Syst. 11, 203 (1966)

30. J.C.C. McKinsey, Isomorhism of games, and strategic equivalence (Princeton University Press, 2016), pp. 117130

31. J. Gabarro, A. Garcia, M. Serna, Theor. Comp. Sci. 412, 6675 (2011)

32. B. Király, G. Szabó, Front. Phys. 8, 59 (2020)

33. D. Fudenberg, J. Tirole, Game Theory (MIT Press, Cambridge, MA, 1991)

34. K.G. Binmore, Fun and Games: A Text on Game Theory (Heath \& Co, Lexington, MA, 1992)

35. W. Poundstone, Prisoner's Dilemma (Doubleday, New York, 1992)

36. M.J. Osborne, A. Rubinstein, A Course in Game Theory (MIT Press, Cambridge, MA, 1994)

37. K. Sigmund, The Calculus of Selfishness (Princeton University Press, Princeton, NJ, 2010)

38. G. Bonanno, Game Theory (Open Access Textbook, 2015)

39. J. Schnakenberg, Rev. Mod. Phys. 48, 571 (1976)

40. J. Friedman, Rev. Econ. Stud. 38, 1 (1971)

41. D. Fudenberg, E. Maskin, Econometrica 54, 533 (1986)

42. L.S. Shapley, R.N. Snow, Ann. Math. Stud. 24, 27 (1950)

43. S. Karlin, Matrix Games, Programming, and Mathematical Economics, Mathematical Methods and Theory in Games, Programming, and Economics, vol. 1 (Pergamon, London, UK, 1959)

44. J. Szép, F. Forgó, Introduction to the Theory of Games, Mathematics and Its Applications, East European Series, vol. 3 (D. Reidel, Dordrecht, 1985)

45. G. Szabó, I. Borsos, Phys. Rep. 624, 1 (2016)

46. J. Ashkin, E. Teller, Phys. Rev. 64, 178 (1943)

47. J. Sólyom, Fundamentals of the Physics of Solids: Structure and Dynamics, vol. I (Springer, Berlin, 2007)

48. A. Aharony, O. Entin-Wohlman, Introduction to Solid State Physics (World Scientific, London, 2018)

49. B. Király, G. Szabó, Phys. Rev. E 96, 042101 (2017)

50. F.Y. Wu, K.Y. Lin, J. Phys. C: Solid State Phys. 7, L181 (1974)

51. F.J. Wegner, J. Phys. C: Solid State Phys. 5, L131 (1972)

52. R.M. Liu, W.Z. Zhuo, J. Chen, M.H. Qin, M. Zeng, X.B. Lu, X.S. Gao, L.M. Liu, Phys. Rev. E 96, 012103 (2017) 
53. G. Szabó, I. Borsos, E. Szombati, Physica A 521, 416 (2019)

54. G. Szabó, I. Borsos, B. Leitner, Phys. Rev. E 100, 032301 (2019)
55. Y. Kang, Q. Pan, X. Wang, M. He, Physica A 392, 2652 (2013)

56. J. Vukov, A. Szolnoki, G. Szabó, Phys. Rev. E 88, 022123 (2013) 